Recepción: 20 / 04 / 2017

Aceptación: 20 / 05 / 2017

Publicación: 15 / 06 / 2017

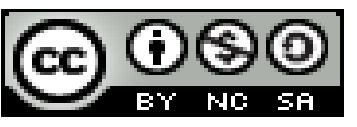

Ciencias Económicas

Artículo de Investigación

\title{
¿Cómo se construye una regulación económica? Caso empírico: Liberalización del sistema financiero en Singapur
}

How is economic regulation built? Empirical case: Liberalization of the financial system in Singapore

Como econômica regulação é construída? caso empírico: Liberalização do sistema financeiro em Cingapura

\author{
Ligia J. Fariño-Maldonado ${ }^{\mathrm{I}}$ \\ ligia.farinom@ug.edu.ec \\ Rubén D. Saldaña-Jara II \\ ruben.saldanaj@ug.edu.ec \\ Ángel G. Salazar-Bustos III \\ angel.salazarb@ug.edu.ec
}

Correspondencia: ligia.farinom@ug.edu.ec

Magister en Tributación y Finanzas; Diploma Superior en Pedagoga Universitaria; Diploma Superior en Tributación; Economista; Universidad de Guayaquil, Guayaquil, Ecuador.

II. Magister en Docencia y Gerencia en Educación Superior; Economista; Universidad de Guayaquil, Guayaquil, Ecuador.

III. Magister en Finanzas y Proyectos Corporativos; Diploma Superior en Diseño Curricular por Competencias; Diploma Superior en Autoevaluación con Fines de Acreditación; Economista; Universidad de Guayaquil, Guayaquil, Ecuador. 


\section{Resumen}

El presente artículo trata de explicar cómo los Gobiernos construyen regulaciones económicas dentro de los países, basados en un marco teórico sobre los diferentes tipos de regulaciones: del Estado, civil, mixta y de riesgo. Se presenta el caso de la liberalización del sistema financiero en Singapur, partiendo de la Crisis Asiática de 1997 como antecedente. Hasta el año 1997 Singapur era una economía en crecimiento, a partir de ese año la Autoridad Monetaria de Singapur (MAS), tomó medidas para liberar el mercado y así evitar que la crisis afecte al crecimiento que se tenía hasta ese momento. La construcción de la regulación tuvo como principal actor al Gobierno de Singapur junto con los representantes del sector financiero. El tipo de regulación implementada fue de riesgo, justamente para mitigar los riegos de una crisis financiera que afectarían el funcionamiento del mercado, pero también se decidió tomar otro tipo de riesgos al liberalizar el sector financiero, con el objetivo de atraer innovación al mercado. También se encontró el tipo de regulación mixta, pero esta se cumple en cierta parte, debido a que actores externos al Gobierno apoyaron con sus criterios en la construcción de la regulación. Finalmente se concluye que la liberalización del sistema financiero bajo un correcto marco de regulación, buscando un equilibrio entre regulación y libertad, permite neutralizar los efectos de una crisis financiera y generar un crecimiento económico sostenible.

Palabras claves: Regulaciones económicas; Singapur; crisis financiera. 


\begin{abstract}
The present article tries to explain how the governments construct economic regulations within the countries, based on a theoretical framework on the different types of regulations: of the State, civil, mixed and of risk. The case of the liberalization of the financial system in Singapore is presented, starting from the Asian Crisis of 1997 as antecedent. Until 1997, Singapore was a growing economy. Since then, the Monetary Authority of Singapore (MAS) has taken steps to liberalize the market and thus prevent the crisis from affecting the growth until then. The construction of the regulation had as main actor the Government of Singapore along with the representatives of the financial sector. The type of regulation implemented was risky, precisely to mitigate the risks of a financial crisis that would affect the functioning of the market, but also decided to take other risks by liberalizing the financial sector, with the aim of attracting innovation to the market. The type of mixed regulation was also found, but this is fulfilled to a certain extent, because external actors to the Government supported with their criteria in the construction of the regulation. Finally, it is concluded that the liberalization of the financial system under a proper regulatory framework, seeking a balance between regulation and freedom, can neutralize the effects of a financial crisis and generate sustainable economic growth.
\end{abstract}

Key words: Economic regulations; Singapore; financial crisis. 


\section{Resumo}

O presente artigo trata de explicar como os Gobiernos construire regulamentos económicos dentro dos países, basados em um marco teórico sobre os diferentes tipos de regulamentos: do Estado, civil, mixta y de risco. Se apresenta o caso da liberalização do sistema financeiro em Singapura, partiendo da crise de Asiática de 1997 como antecedente. Até o ano de 1997 Cingapura era uma economia em crescimento, a partir desse ano, a Autoridade Monetária de Singapura (MAS), tomó medidas para liberar o mercado e, portanto, evitar a crise afecta ao crescimento que está em pé até esse momento. A construção da regulação ocorreu como principal ator no governo de Singapura juntamente com os representantes do setor financeiro. O tipo de regulação implementada foi de risco, justamente para mitigar os riegos de uma crise financeira que afetou o funcionamento do mercado, mas também se decidió tomar outro tipo de riscos ao liberar o setor financeiro, com o objetivo de atraer inovação no mercado. Também se encontrou o tipo de regulação mista, mas esta se cumprido em cierta parte, devido a que os órgãos externos foram apoiados com seus critérios na construção da regulação. Finalmente, conclui que a liberalização do sistema financeiro sob um marco de regulação correto, buscando um equilíbrio entre regulação e liberdade, permita neutralizar os efeitos de uma crise financeira e gerar um crescimento económico sustentável.

Palavras-chave: Regulamentações econômicas; Cingapura; Crise financeira. 


\section{Introducción.}

En la actualidad existe un amplio debate sobre las conveniencias de liberalizar o no el sistema financiero dadas las diferentes crisis financieras. La política implementada por un Gobierno debe procurar que los mercados sean resistentes y eficientes, tanto como sea posible, por tal motivo, los responsables de diseñarla deben deshacerse de las dificultades tradicionales con respecto a la sobrerregulación, sobreimposición y sobreprotección, permitiendo a los mercados funcionar por ellos mismos. (Lindsey, 1993)

En el presente trabajo se analiza el caso empírico de Singapur, donde posterior a la Crisis Financiera Asiática de 1997, se aplicó una regulación para liberalizar el sistema financiero y de esta manera también contrarrestar los efectos de esta crisis. Se realiza el análisis de este caso debido a la relevancia que puede tener para el Ecuador, ya que liberalizar el sistema financiero podría ser una forma de contrarrestar una eventual crisis económica, esto también deja importantes lecciones para el país, entre esas, incluir a más sectores en la toma de decisiones gubernamentales. El desarrollo del presente documento está dividido en cuatro secciones: en primer lugar se analiza cómo empezó esta crisis financiera y de qué manera afectó a los países de la región, enfocándonos en el caso de Singapur. Como segundo punto se expone las recomendaciones a partir de las cuales el Gobierno de Singapur tomó medidas para liberalizar el sistema financiero del país. En el tercer punto se describen los tipos de regulaciones identificando el que corresponde al presente caso, presentando los actores involucrados y su participación. Finalmente se concluye que la liberalización del sistema financiero, bajo un apropiado marco de regulación y libertad, contribuye a un crecimiento económico sostenible. 


\section{Materiales y métodos.}

La regulación es el conjunto de diversos instrumentos mediante los cuales los Gobiernos pueden establecer normas a empresas y ciudadanos. (León Bolaños \& Barrionuevo, 2015) El desarrollo de una regulación está relacionado al papel del gobierno en la sociedad y en el mercado. La literatura teórica sobre la regulación es a la vez empírica (tratar de comprender, describir y explicar casos de estudio o investigación); normativa (debatir sobre lo que los gobiernos deberían o no deberían hacer de acuerdo a un conjunto de valores); y prescriptiva (ofrecer soluciones para los problemas sociales o correcciones a las fallas del mercado). (Krawchenko, 2012) El principal entendimiento que se tiene sobre regulación es una forma de "Comando y control" la cual es una regulación desde el Estado a través del uso de reglas y su incumplimiento causa sanciones. Se asume que es una relación unilateral, es decir el Gobierno dictamina y otros lo cumplen. Pero los instrumentos usados por el Gobierno son inapropiados y a veces no sofisticados, ya que este no tiene el suficiente conocimiento sobre las causas de los problemas y no pueden diseñar soluciones apropiadas para estos. (Black, 2002) La propia cultura de cada país engloba un sistema económico, institucional, jurídico y político, y esta influencia de las fuerzas culturales o sociales participan en las regulaciones o desregulaciones del país. (Hancher \& Moran, 1989) Así mismo algunos manifiestan que la regulación también es realizada por actores no gubernamentales como por ejemplo: organizaciones, asociaciones, empresas, individuos, comités técnicos y otros organismos especializados. (Black, 2002) Entonces las regulaciones en ese caso reflejan un trabajo en conjunto o mixto, es decir entre el Gobierno y los diferentes organismos dentro de un país.

El riesgo necesita ser controlado, pero el hecho de tomar riesgos constituye un elemento fundamental en la dinámica de la economía y de una sociedad innovadora. Vivir en un mundo globalizado significa encontrarse con varias situaciones de riesgo. (Giddens, 2000) Algunas 
¿Cómo se construye una regulación económica? Caso empírico: Liberalización del sistema financiero en Singapur

regulaciones se pueden introducir con fines relacionados a controlar el riesgo, tales como la creación de un entorno propicio para la inversión o la facilitación del comercio. Una buena gestión de riesgos hace más que ayudar a evitar catástrofes y proporcionar seguridad y tiene como objetivo evitar pérdidas innecesarias, evitables e inesperadas. (Jachia \& Nikonov, 2012)

\section{Caso Empírico: La liberalización del sistema financiero en Singapur}

El 2 de julio de 1997 con la devaluación del baht tailandés empezó una etapa de gran deterioro en los mercados financieros de Asia. (Corsetti, Pesenti, \& Roubini, 1999) (Vilariño, 2000) Estos países experimentaron un fuerte descenso en sus monedas, bolsa de valores y otros activos financieros, además de que la crisis amenazaba sus sistemas financieros y causó una contracción en la actividad económica. (Chen \& Paulraj, 2004) Muchas han sido las causas y soluciones sugeridas para frenar esa crisis (Berg \& Pattillo, 1999) (International Monetary Fund. Research Dept, 1998) Lo que comenzó como una crisis monetaria pronto afectó a la economía en general y termino como crisis económica en algunos países.

Hasta el año 1997 Singapur era una economía pujante y uno de los cuatro "Tigres Asiáticos" con una tasa de crecimiento anual del PIB de 6,26\% en promedio desde que logró su independencia en 1965. (The World Bank Working for a World Free of Poverty, 2015) Singapur no se vio afectada directamente, sino que sufrió los efectos indirectos de la desaceleración económica de sus vecinos de la región. Al darse cuenta de que la estimulación de la demanda interna no era una opción viable en una recesión causada por circunstancias externas, el Gobierno de Singapur debió aplicar varias medidas para ayudar a aliviar la carga de los costes de las empresas y los individuos. (Chew, 2009)

Justo antes de que el baht tailandés se desplome en julio de 1997, éste ya había sido objeto de intensos ataques especulativos. Durante un tiempo, el gobierno de Tailandia logró proteger la 
moneda, que había sido vinculado al dólar de Estados Unidos (EEUU). Sin embargo, se anunció que ya no intervendría, permitiendo que el baht flote y ese mismo día comenzó la fuerte depreciación de la moneda local frente al dólar estadounidense. (Laplamwanit, 1999) Esto causó de inmediato el pánico entre los inversores, y otras monedas de la región, tales como el peso filipino, rupia indonesia y el ringgit de Malasia. Pronto, los inversores extranjeros perdieron la confianza en las monedas y las economías de estos países del Este Asiático. (Dornbusch, Park, \& Claessens, 2000)

Teniendo en cuenta sus estrechos vínculos económicos con otros países de Asia, Singapur no podía escapar de los efectos de la crisis financiera que enfrentaron sus vecinos de la región a pesar de la fuerte economía que tenía hasta ese momento. Muchas empresas quebraron y dejaron a sus trabajadores sin empleo, entonces para poder sobrevivir, otras empresas redujeron sus costes al recortar el personal, suspendieron nuevas contrataciones y bajaron los salarios de sus trabajadores, es así que en el último trimestre de 1997, 4.280 trabajadores fueron despedidos, más de tres veces la cantidad en comparación con el trimestre anterior. (Ministry of Information and The Arts, 1998) Mientras que para 1998 la situación se agravó con reducciones trimestrales promedio de unos 7.300 trabajadores (Ministry of Manpower Singapore, 2016). El cierre de empresas y la reducción del personal también se tradujeron en una menor demanda de propiedades comerciales, industriales y residenciales, que pusieron presión a la baja de los precios de los inmuebles y alquileres. Los precios de las acciones cayeron y esto tuvo un efecto negativo en la economía, lo que debilitó aún más la confianza del consumidor (Economic survey of Singapore, 1999).

El sector de los servicios financieros, por otra parte, fue uno de los más afectados y donde se registró una fuerte contracción: 7,4\% en 1998 (World Bank, 2017b). Reflejando un panorama de una economía debilitada en Singapur hasta ese momento, además causando el deterioro de la confianza en las economías regionales y el hecho de que los bancos locales frenaron sus actividades de 
¿Cómo se construye una regulación económica? Caso empírico: Liberalización del sistema financiero en Singapur

préstamo y en general prestaban menos a las empresas y los individuos. (Radelet \& Sachs, 1998) En general, la economía de Singapur se contrajo un 1,4\% en 1998 en términos de producto interno bruto (PIB). El impacto de la crisis en Singapur fue indirecto, pero tuvo un gran alcance.

A pesar de que Singapur tenía una economía fuerte, los efectos de la crisis también llegaron a afectarlo, ante este panorama era necesario que se tomen medidas que contrarresten estos efectos a corto plazo, y a su vez minimizar el riesgo de que el país entre en una crisis más profunda (afectando a otros sectores de la economía) como otros países asiáticos. Tanto el Gobierno como sus reformas implementadas contaban con una gran aceptación por parte de la sociedad, entonces bajo este escenario era un momento adecuado para tomar medidas y controlar esta crisis. (Worldwide Governance Indicators, 2016)

Frente a este panorama de crisis el gobierno de Singapur implementó una serie de medidas en 1998 con el objetivo principal de liberalizar el mercado y así evitar que la crisis afecte el crecimiento que se tenía hasta ese momento, convirtiéndose en un centro financiero internacional clave en la región. (Chia Siow, 1998) Desde aquí el Gobierno comienza a ejercer su rol dentro de esta regulación, ya que un Comité de Finanzas Corporativo es formado por el Grupo de Revisión del Sector Financiero para que realice recomendaciones. (Monetary Autorithy of Singapore, 2016a)

Para lograr su objetivo y aprovechando la alta aceptación por parte de la ciudadanía (Quah, 2010) el Gobierno utilizó mecanismos mediante los cuales ejerció su autoridad e interactuó con las Instituciones. Esto pudo ser posible mediante la publicación de un documento tipo consulta por parte del Comité de Finanzas Corporativo, donde se presentaba un resumen de sus puntos de vista preliminares y buscaba comentarios sobre los mismos. (Corporate Finance Committee, 1998a) 
En esta etapa de consulta se buscaba la participación de diversos agentes del sistema financiero como Bancos y Bolsa de Valores, es decir sociedades cotizadas e inversores.

El Comité detalló los términos, los cuales se tomaron como punto de partida para diseñar las medidas a ser implementadas, el fin de estos términos era elaborar un marco apropiado con normas, reglamentos y directrices administrativas que permitan regular la captación de fondos, que apoyen el crecimiento constante y sostenible de las empresas en el país, que fomenten la innovación y el emprendimiento, siendo el punto clave posicionar a Singapur como un centro atractivo para la actividad financiera internacional y de esta manera captar fondos. Otro punto importante era recomendar nuevas capacidades a las empresas para que puedan satisfacer sus necesidades de financiación como su deuda, mediante un marco de normas y reglamentos. Por último se debía recomendar, a consideración del Gobierno, las funciones adecuadas que debían ejercer la Bolsa de Valores de Singapur ("SES"), la Autoridad Monetaria de Singapur ("MAS") y el Registro de Empresas y Negocios ("RCB") y otras agencias gubernamentales (Monetary Autorithy of Singapore, 2016b).

Al hacer sus recomendaciones finales, el Comité tuvo en cuenta todos los puntos de vista. Las medidas que finalmente tomó tenían que ver con desarrollar las actividades de la banca aún con más ímpetu atrayendo grupos de bancos extranjeros y brindarles el apoyo profesional y tecnológico que estos requerían, hacer que estos sitúen sus sedes en el mercado de Singapur y facilitar su membresía a la SES. Otra medida fue el desarrollo del sector de capital de riesgo y de esta manera atraer inversores con experiencia, que fomenten la creación de empresas que coticen y que a su vez se les ofrezca una amplia gama de servicios; también hacer de Singapur un centro de emprendimiento regional para las inversiones de capital. Una medida para fomentar la innovación en la industria financiera fue que se quiten los obstáculos a ésta y además se debía hacer una 
¿Cómo se construye una regulación económica? Caso empírico: Liberalización del sistema financiero en Singapur

revisión del entorno fiscal en Singapur para hacerlo más competitivo. Para lograr una mejor relación con el sistema financiero internacional se debía acelerar la adopción de tecnologías para facilitar la distribución de información y el comercio. Así mismo como punto de partida Internet debía ser aceptado como medio para la distribución de información e incluso estaban dispuestos a cambiar la ley respecto a esto (Corporate Finance Committee, 1998a).

El Gobierno aceptó la mayoría de las recomendaciones del Comité de Finanzas Corporativo de estudio para liberalizar el sector financiero (Monetary Authority of Singapore, 1998).

A principios de 1999, la economía de Singapur ya estaba mostrando signos de recuperación. En el primer trimestre de 1999, la economía volvió a un crecimiento positivo, impulsado por una fuerte recuperación en el sector manufacturero. La recuperación se mantuvo durante todo el año y el PIB global para todo el año 1999 creció un 7,2\%, muy superior a la previsión inicial del gobierno de entre $-1 \% y+1 \%$. (World Bank, 2017c).

Según (Loong, 2004) Singapur tiene ahora uno de los entornos bancarios más liberales de Asia. La liberalización de la banca ha logrado hasta ahora su objetivo de lograr una mayor competencia. Se ha fomentado la consolidación de los bancos locales. Las medidas de liberalización y reformas de los últimos años han puesto al sector financiero sobre una base firme. (Hua \& Randhawa, 2006) Pero igual se debe identificar y responder a nuevos desafíos y oportunidades, con el fin de mantener el sector financiero dinámico y competitivo.

\section{Análisis del caso empírico}

Bajo este contexto y analizada la teoría, el reto que tenía Singapur era el de implementar medidas efectivas que reduzcan el impacto de la crisis, en aquel momento la crisis era lo que 
representaba el riesgo, ya que ésta afectaría la actividad económica del país (en los últimos años había estado en un constante crecimiento) y por ende como toda crisis podría provocar pánico financiero, además de que ya se habían visto afectados otros países de la región (Iriana y Sjoholm, 2002; Glassman, 2001; Baig y Goldfajn, 1999). Mientras tanto las reformas son el medio, instrumento o mecanismo utilizado para lograr mitigar o controlar los efectos de la crisis (riesgo). Estas reformas tuvieron como base principal la liberalización del sistema financiero, que a su vez modificaba y moldeaba el comportamiento de las personas y empresas, ya que la interacción de las fuerzas del mercado resulta ser más eficiente para modificar el comportamiento de los bancos, de lo que en su lugar las fuertes regulaciones por parte del Estado pretenderían serlo (Jordan, 1994), esta sería la forma en que buscaba convertirse en una economía más abierta al comercio internacional y tener un fuerte desarrollo financiero.

El Gobierno, representado por el MAS, siendo el actor fundamental en la elaboración de estas medidas, necesitaba la colaboración de los representantes del sector financiero para ser capaz de convertir a Singapur en un centro financiero internacional, ya que para lograr este objetivo necesitaba el talento y las habilidades de la industria financiera (Monetary Authority of Singapore, 1999). Durante la elaboración de la regulación, tanto las instituciones financieras, específicamente el sector bancario, como la Bolsa de Valores, fueron fundamentales con su participación, que se dio mediante la creación del Financial Centre Advisory Group (FCAG) el cual estaba integrado por los principales miembros de la comunidad financiera de Singapur y el International Advisory Panel (IAP) formado por banqueros y financieros distinguidos, el MAS buscaba aprovechar la experiencia de quienes integraban estos grupos y así dar sus recomendaciones. El objetivo del IAP y FCAG fue aportar comentarios sobre asuntos de regulación y supervisión, además de apoyar el desarrollo y facilitar el intercambio de puntos de vista con los participantes del sector financiero en Singapur 
¿Cómo se construye una regulación económica? Caso empírico: Liberalización del sistema financiero en Singapur

(Monetary Authority of Singapore, 2001). Por esto fue que el Comité solicitó sus opiniones mediante el documento tipo consulta a estos grupos para tener un panorama más amplio de la situación, ya que con su experiencia y conocimientos contribuirían al Gobierno a tomar las medidas adecuadas y lograr cumplir con su objetivo.

El caso de Singapur demuestra que parte importante de la teoría sobre la regulación de riesgo se cumple, primero porque las regulaciones fueron usadas para contrarrestar los efectos de una crisis que alteraba el funcionamiento del mercado, evitando un riesgo que el Gobierno de Singapur no quería asumir. Así mismo se destaca la decisión de tomar otro tipo de riesgos al liberalizar el sector financiero, con el cual buscaban traer innovación al mercado y fue justamente lo que sucedió ya que son catalogados como un importante centro financiero de la región. También se encontró dentro del caso el tipo de regulación mixta, pero señalando que esta se cumple en parte, aunque varios actores externos al Gobierno apoyaron con sus criterios en la construcción de la regulación, finalmente fue el Gobierno quien adoptó las recomendaciones y decidió implementarlas como medidas.

\section{Conclusiones.}

En este artículo se ha revisado cómo se construyó la regulación que permitió la liberalización del sistema financiero y se demostró cómo esta ayudo a frenar la crisis financiera para el caso de Singapur.

Antes de la crisis, Singapur tenía un ritmo de crecimiento importante, siendo uno de los 4 "tigres asiáticos", cuando estalló la crisis comenzó una etapa de deterioro en los mercados financieros asiáticos. Ante este panorama era necesario que se tomen medidas para contrarrestar los efectos de la crisis que terminaron afectando en gran escala a países cercanos como: Tailandia, Malasia, Indonesia, Filipinas y Corea del Sur, etc. Por su parte Singapur no resultó ser de los más 
afectados por esta crisis (Garcia y Olivié 1999). La rápida respuesta del Gobierno ante esta situación fue reunir al Comité de Finanzas Corporativo, el cual propuso unos términos como base para implementar las medidas que se tomaron, además de un documento tipo consulta donde se buscaba participación de diferentes representantes del sector financiero, y las recomendaciones finales las hizo el Comité al Gobierno de Singapur. El objetivo era liberalizar el sistema financiero y volverlo un centro financiero internacional, siendo esta su postura para controlar la crisis.

Liberalizar el sistema financiero permitió a Singapur frenar esta crisis y desarrollarse financieramente, entonces esto implica que la liberalización del mercado bajo un marco adecuado de regulación/supervisión (buscando equilibrio entre regulación y libertad) permite contrarrestar/frenar los efectos de una crisis financiera y generar un mayor crecimiento económico.

El análisis de este caso deja importantes lecciones para el Ecuador, ya que la actual situación económica no es la mejor y las proyecciones tampoco parecen ser alentadoras. Cabe recalcar que en Ecuador en los últimos años no se ha incluido al sector privado en las decisiones gubernamentales, de cara al futuro esto debería cambiar. Los encargados de las políticas económicas del país podrían tomar como ejemplo lo realizado por Singapur para implementar futuras regulaciones económicas que permitan liberalizar al sector financiero. Una liberalización financiera bajo una correcta regulación, contribuye a aliviar los efectos de una crisis; además del Gobierno, se debería incluir en las decisiones que afecten al sector financiero a todos los organismos involucrados directa o indirectamente, ya que pueden contribuir, desde su campo de especialización, a construir regulaciones que beneficien al país. 


\section{Bibliografía.}

Berg, A., \& Pattillo, C. (1999). Are Currency Crises Predictable? A Test. IMF Staff Papers, 46(2).

Black, J. (2002). Critical reflections on regulation. London: London School of Economics and Political Science.

Chen, I., \& Paulraj, A. (2004). Towards a theory of supply chain management: the constructs and measurements. Journal of Operations Management, 22(1), 119-150.

Chew, V. (2009). Asian financial crisis, 1997-1998. Singapore: National Library Board Singapore.

Chia Siow, Y. (1998). The Asian Financial Crisis: Singapore's Experience and Response. Osean Economic Bulletin, 15(3), 297-308.

Corsetti, G., Pesenti, P., \& Roubini, N. (1999). What caused the Asian currency and financial crisis? Cambridge: National Bureau of Economic Research.

Dornbusch, R., Park, Y., \& Claessens, S. (2000). Contagion: How it spreads and How it can be stopped. Forthcoming World Bank Research Observer.

Glassman, J. (2001). "Economic Crisis in Asia: The Case of Thailand”. Economic Geography, Vol. 77, núm. 2 (Abr., 2001), pp. 122-147

Giddens, A. (2000). Runaway World. New York: Library of Congress Cataloging in Publicatiion Data.

Hancher, L., \& Moran, M. (1989). Introduction: Regulation and deregulation. Europena Journal of Political Research, 17(2), 129-136.

Hua, L., \& Randhawa, D. (2006). The Banking Sectors in Hong Kong and Singapore: What Has Liberalization Wrought? An X-Efficiency Study. Saw Centre for Financial Studies. Working Paper, $6(3)$.

International Monetary Fund. Research Dept. (1998). The Macroeconomic Impact of Privatization. IMF Staff papers, 45(2).

Iriana, R., Sjoholm, F. (2002). "Indonesia's economic Crisis: Contagion and Fundamentals" The Developing Economies, XL-2 (Junio 2002) 135-51.

Jachia, L., \& Nikonov, V. (2012). Risk Management in Regulatory Frameworks: Towards a Better Management of Risks. Washington D. C.: United Nations.

Krawchenko, T. (2012). Institutional Solutions to Jurisdictional Fragmentation: The Implications of Regional Special Purpose Bodies for how City-regions are Governed in Canada. Ottawa: Carleton University.

Laplamwanit, N. (1999). A Good Look at the Thai Financial Crisis in 1997-98. Nueva York: Columbia University.

León Bolaños, N., \& Barrionuevo, M. (2015). Propuesta de Instrumentos de Política Pública que promuevan la protección y cuidado ambiental en la gestión empresarial del Ecuador. Quito: Pontificia Universidad Católica del Ecuador. 
Lindsey, L. (1993). Economic Challenges of the 1990s. Cato Journal, 13(2), 165-169.

Loong, L. (2004). Financial Sector - Liberalisation and Growth. Monetary Authority of Singapore.

Ministry of Information and The Arts. (1998). Annual Economic survey of Singapore. Singapore: National Archives of Singapore.

Quah, J. (2010). Trust and governance in the Philippines and Singapore: a comparative analysis. International Public Management Review.

Radelet, S., \& Sachs, J. (1998). The onset of the East Asian Fianancial Crisis. Cambridge: National Bureau of Economic Research.

The World Bank Working for a World Free of Poverty. (2015). Singapore, World Bank Group Respond to Global Demand and Establish Major Infrastructure and Urban Development Hub. Singapore: MOF.

Vilariño, A. (2000). La crisis financiera asiática. Madrid: Publicaciones Ángel Vilariño. 